\title{
A Simplified Method for Calculating Spectral Emission of Nonequilibrium Air Plasmas in Hypersonic Shock-Layers
}

\section{Xin He}

Department of physics, National University of Defense Technology

\section{Cheng Gao}

Department of physics, National University of Defense Technology

Tao Jiang ( $\square$ fengqiaoren999@163.com )

Computational Aerodynamics Institute of CARDC

\section{Research}

Keywords: Spectral emission, Nonequilibrium, Air plasma, Hypersonic

Posted Date: December 14th, 2020

DOl: https://doi.org/10.21203/rs.3.rs-122824/v1

License: (c) (1) This work is licensed under a Creative Commons Attribution 4.0 International License.

Read Full License 


\title{
A simplified method for calculating spectral emission of nonequilibrium air plasmas in hypersonic shock-layers
}

\author{
Xin He, ${ }^{1}$ Cheng Gao, ${ }^{1}$ and Tao Jiang ${ }^{2, *}$ \\ 1 Department of Physics, National University of Defense Technology, Changsha 410073, China \\ 2 Computational Aerodynamics Institute, China Aerodynamics Research and Development Center, \\ Mianyang 621000, China \\ * Corresponding author. E-mail: fengqiaoren999@163.com
}

\begin{abstract}
A simplified method for calculating the spectral emission of nonequilibrium air plasmas is developed. In order to obtain the nonequilibrium energy level populations, the nonequilibrium coefficients are introduced into the Saha-Boltzmann equation. These nonequilibrium coefficients are calculated by using several significant radiative processes. An approach to the determination of nonequilibrium electronic energy level populations of diatomic molecules is also presented. Based on the method, spectral emission of atoms and molecules in a typical air plasma cell is investigated. The results reveal that there is a significant difference between the nonequilibrium and equilibrium emission. We apply the method to the nonequilibrium AVCO R-156 experiment. Good agreement with the measured data is shown, indicating that the method is reasonable and has good accuracy.
\end{abstract}

Key Words: Spectral emission, Nonequilibrium, Air plasma, Hypersonic

\section{Introduction}

During the hypersonic reentry, a significant portion of the heating experienced by a vehicle may be due to the emission of air plasmas in the shock-layer. To investigate the radiative heating, it is highly important to calculate the spectral emission of air plasmas [1].

The air plasmas in the shock-layer may be equilibrium or nonequilibrium [2,3]. For equilibrium air plasmas, their spectral emission can be easily calculated by means of equilibrium statistical and spectral theory. However, for nonequilibrium air plasmas, the prediction of their emission is a challenge.

For this reason, most of the physical models and computational codes in the literature have tried their best to address nonequilibrium radiation [4-10]. Full models, such as the-collisional-radiative-(CR)-model,-are-well-known-to-be-accurate-[11,12]-However, their-computa tional-cost-is-very-high.-To-improve-the-computational-efficiency, researchers-tend-to-develop-simpli fied-models.-These-simplified-models will-be-desirable-and-useful-in-engineering.-The-nonequilibrium -air-radiation-(NEQAIR)-code-presented-by-Park-is the-most-widely-used.-Although-NEQAIR-empl oyed the-so-called-quasi-steady-state-(QSS)-assumption-[13,14], its-computational-cost-is-not-low-du eto-the-consideration-of-many-collisional-and-radiative-processes-in-air plasmas-[15].

In this paper, based on the main idea of the CR model, a simplified method for calculating

The work is supported by Nanational Numerical Windtunnel 
nonequilibrium air plasma radiation is developed. The method uses a modified Saha-Boltzmann equation to represent the nonequilibrium energy level population. By solving the conservation equation containing significant radiative processes, the nonequilibrium population of bound electronic energy levels can be obtained. Based on a typical air plasma cell, the difference between the nonequilibrium and equilibrium emission of particles is analyzed. In addition, application of the method in nonequilibrium AVCO R-156 experiments is discussed.

\section{Simplified method}

In the following, we consider the 11-species air model that contains $\mathrm{N}, \mathrm{N}^{+}, \mathrm{O}, \mathrm{O}^{+}, \mathrm{N}_{2}, \mathrm{~N}_{2}^{+}, \mathrm{O}_{2}$, $\mathrm{O}_{2}^{+}, \mathrm{NO}, \mathrm{NO}^{+}$and free electrons. The method for determining the bound electronic energy level populations is first explained, which is based on our previous work [16,17]. Then, we apply the method to some typical plasmas and analyze the nonequilibrium radiation from atoms and molecules, respectively.

\subsection{Bound electronic energy level populations}

The main idea of the full CR model is that the population of each bound electronic energy level is affected by various collisional and radiative processes [1]. If the QSS assumption is used, the population of the $i$ th bound electronic energy level $n_{i}$ is determined by

$$
\left.\frac{d n_{i}}{d t}\right|_{\text {collisional }}+\left.\frac{d n_{i}}{d t}\right|_{\text {radiative }}=0
$$

In Eq. (1), the collisional processes can be subdivided into cases where the impacting particles are electrons, ions, or neutral particles. The radiative processes include radiative transition, radiative recombination, and so on $[1,12]$. The full CR model is accurate because it considers as many processes as possible. However, this leads to a large number of coupled rate-equations and a huge increase in the computational cost.

For general hypersonic air plasmas, the departure from thermal equilibrium is usually not very large. By introducing a correction factor into the Saha-Boltzmann equation [16,17], there will be

$$
n_{i}=b_{i} n_{e} n^{+} \frac{g_{i}}{2 Q^{+}}\left(\frac{h^{2}}{2 \pi m_{e} k T_{e}}\right)^{3 / 2} \exp \left(\frac{I-E_{i}}{k T_{e}}\right)
$$

where $b_{i}$ is the correction factor for the population of the $i$ th level, $n_{e}$ and $n^{+}$are the number density of free electrons and charged particles, $Q^{+}$is the partition sum of charged particles, $g_{i}$ and $E_{i}$ are the degeneracy and energy of the $i$ th level, $I$ is ionization energy, $T_{e}$ is electronic temperature, $m_{e}$ is electron mass, $h$ is Planck constant, $k$ is Boltzmann constant.

To calculate the $b_{i}$ in Eq. (2), three most significant radiative processes, that is, radiative recombination, spontaneous transitions and photo excitation from the ground state, are considered. After some simplifications are made [16], this leads to 


$$
n_{i} \sum_{j=2}^{i-1} A_{i j} \approx \sum_{j=i+1}^{\infty} n_{j} A_{j i}+n_{e} n_{0}^{+} R_{i}
$$

where $n_{j}$ is the population of the $j$ th level, $A_{i j}$ and $A_{j i}$ are the transition probabilities, $n_{0}^{+}$is the number density of ground-state charged particles, $R_{i}$ is the radiative recombination coefficient of the $i$ th level [1].

Based on Eqs. (2) and (3), $b_{i}$ can be calculated, and then the bound electronic energy level populations are obtained.

Specifically, the ionization in nonequilibrium air plasmas is usually highly insufficient [18]. Thus, for several lowest levels, the Boltzmann distribution characterized by $T_{e}$ can be directly assumed [19], as NEQAIR code did. For several highest levels, the Saha distribution characterized by $T_{e}$ can be used [17]. For the other levels, the population should be calculated by Eqs. (2) and (3).

\subsection{Atomic emission}

When the speed of a vehicle is high in earth reentry, the diatomic species behind the shock may be highly dissociated [20]. In this situation, spectral emission of $\mathrm{N}$ and $\mathrm{O}$ may be dominant [21,22]. Therefore, calculation of atomic radiation is highly important.

We employ Park's electronic level system [2]. The nonequilibrium air plasmas is typically optically thick for radiation below $200 \mathrm{~nm}$. It means that the atomic lines corresponding to the transitions from high levels to levels 1-3 can be totally absorbed. Thus, in Eq. (3), the ground state(s) is not level 1 but are levels 1-3. Equation (3) transforms to

$$
n_{i} \sum_{j=4}^{i-1} A_{i j}=\sum_{j=i+1}^{j_{\max }} n_{j} A_{j i}+n_{e} n_{1}^{+} R_{i}
$$

where $j_{\max }$ equals to 22 (for $\mathrm{N}$ ) or 19 (for O).

In order to further improve the efficiency, it is not necessary to calculate the nonequilibrium population of all levels. Table 1 lists the atomic lines based on NEQAIR [7]. As mentioned above, atomic lines below $200 \mathrm{~nm}$ are almost totally absorbed. For the prediction of radiative heating, the atomic lines above $200 \mathrm{~nm}$ are of highly important. Most of the strong atomic lines above $200 \mathrm{~nm}$ are related to levels 6-12. This suggests that we can just calculate the nonequilibrium population of levels 6-12 based on Eqs. (2) and (3). Since the other lines (related to the levels except levels 6-12) are relatively weak, the use of equilibrium population would have little influence on the prediction of spectral emission or radiative heating.

Table 1 Atomic lines analysis.

\begin{tabular}{cccc}
\hline atoms & total lines & above $200 \mathrm{~nm}$ & strong and related to levels 6-12 \\
\hline $\mathrm{N}$ & 179 & 107 & 87 \\
$\mathrm{O}$ & 109 & 83 & 40 \\
\hline
\end{tabular}


To investigate the difference between the nonequilibrium and equilibrium radiation of atoms, we chose an arbitrary air plasma cell. Table 2 lists the condition of the air plasma cell [9]. In the spectral calculations, we only consider bound-bound transitions of $\mathrm{N}$ and $\mathrm{O}$. For the shape of each emission line, the Voigt profile is assumed [3].

Table 2 The condition of the air plasma cell.

\begin{tabular}{cc}
\hline Parameters & Value \\
\hline translational temperature $T_{t}, \mathrm{~K}$ & 31113 \\
rotational temperature $T_{r}, \mathrm{~K}$ & 18027 \\
vibrational temperature $T_{v}, \mathrm{~K}$ & 12280 \\
electronic temperature $T_{e}, \mathrm{~K}$ & 14888 \\
$\mathrm{~N}, \mathrm{~cm}^{-3}$ & $1.34 \times 10^{16}$ \\
$\mathrm{O}, \mathrm{cm}^{-3}$ & $1.01 \times 10^{17}$ \\
$\mathrm{~N}_{2}^{+}, \mathrm{cm}^{-3}$ & $2.27 \times 10^{16}$ \\
$\mathrm{~N}_{2}, \mathrm{~cm}^{-3}$ & $1.75 \times 10^{19}$ \\
$\mathrm{~N}^{+}, \mathrm{cm}^{-3}$ & $1.59 \times 10^{14}$ \\
$\mathrm{O}^{+}, \mathrm{cm}^{-3}$ & $1.24 \times 10^{14}$ \\
free electrons, $\mathrm{cm}^{-3}$ & $5.02 \times 10^{14}$ \\
\hline
\end{tabular}

Figure 1 shows spectral emission of the two atomic species. In Figs. 1(a) and 1(b), the electronic energy level population of $\mathrm{N}$ and $\mathrm{O}$ is assumed to be Boltzmannian with $T_{e}$. However, in Figs. 1(c) and 1(d), the population of $\mathrm{N}$ and $\mathrm{O}$ is calculated by means of the above-mentioned method. One can immediately find that the characteristics of the spectrum are significantly different. For the emission of N, we draw a comparison between Figs. 1(a) and 1(c). It is shown that the Boltzmannian assumption will cause the emission to be overestimated. For O, a similar phenomenon is seen by a comparison between Figs. 1(b) and 1(d). The results imply that the use of Boltzmannian distribution could lead to a considerable error in the prediction of radiative heating. 

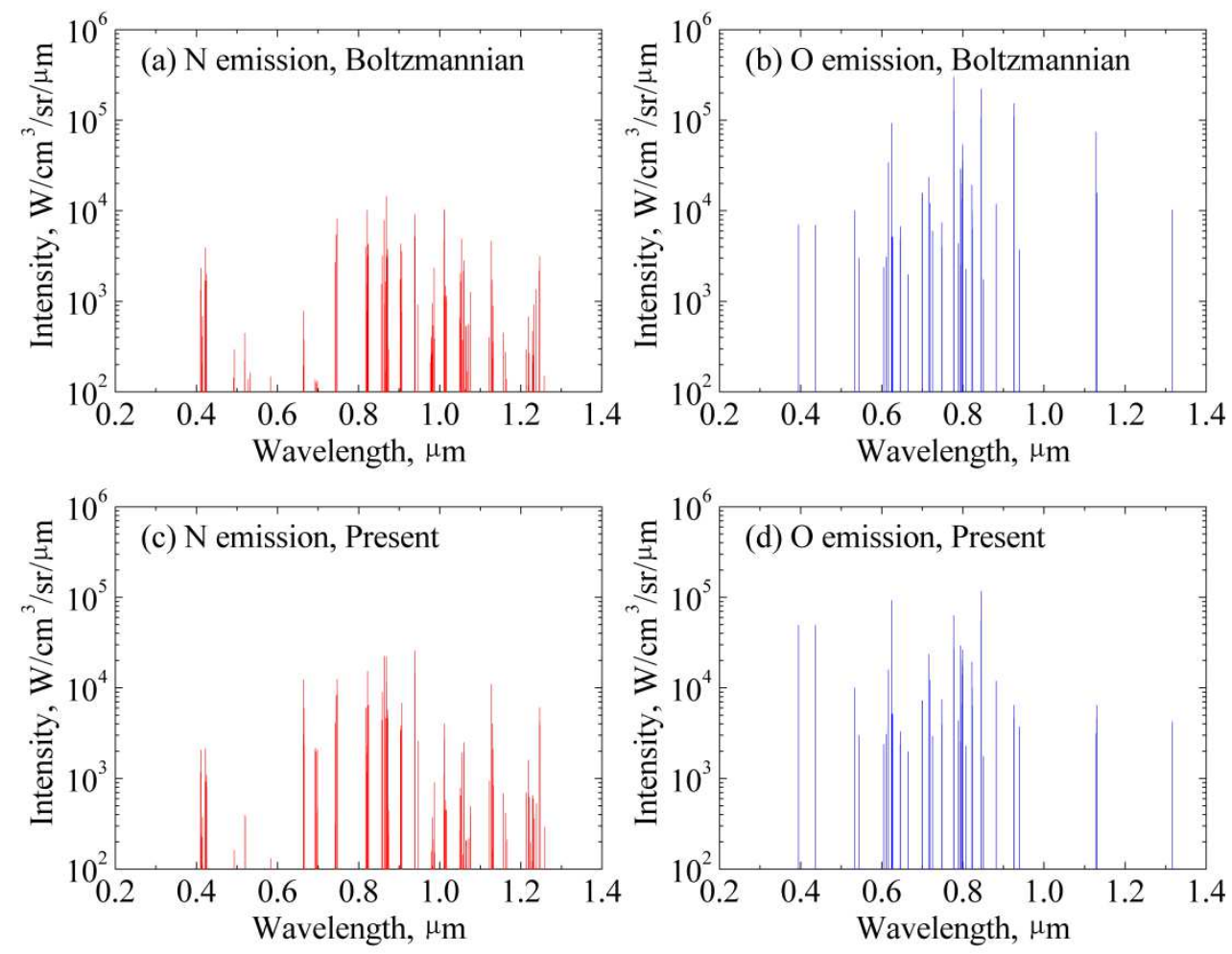

Fig. 1 spectral emission of $\mathrm{N}$ (a) and $\mathrm{O}$ (b) assuming a Boltzmannian distribution with $T_{e}$. Nonequilibrium emission of $\mathrm{N}(\mathrm{c})$ and $\mathrm{O}(\mathrm{d})$ using the present method.

\subsection{Diatomic molecular emission}

For diatomic molecules in air plasmas, the relaxation of electronic energy levels is quicker than vibrational levels and dissociation processes [23]. Based on this, we assumed that the molecules do not experience dissociation during the investigated time step. In order to make use of Eq. (3), we need to determine the radiative recombination coefficient.

We first attempt to found a relationship between the primary quantum number and the ionization energy of a certain electronic level. If the Hydrogen-like approximation is used, this relationship will be

$$
\omega_{i}=I_{H} / I_{i}{ }^{1 / 2}
$$

where $\omega_{i}$ and $I_{i}$ are the primary quantum number and the ionization energy of the $i$ th level, $I_{H}$ is the ionization energy of atomic hydrogen. Considering the important electronic energy levels of diatomic molecules for air plasma radiation, we can obtain Table 3 for the three diatomic molecules [24,25].

Table 3 The values of $\omega_{i}$ for diatomic molecules.

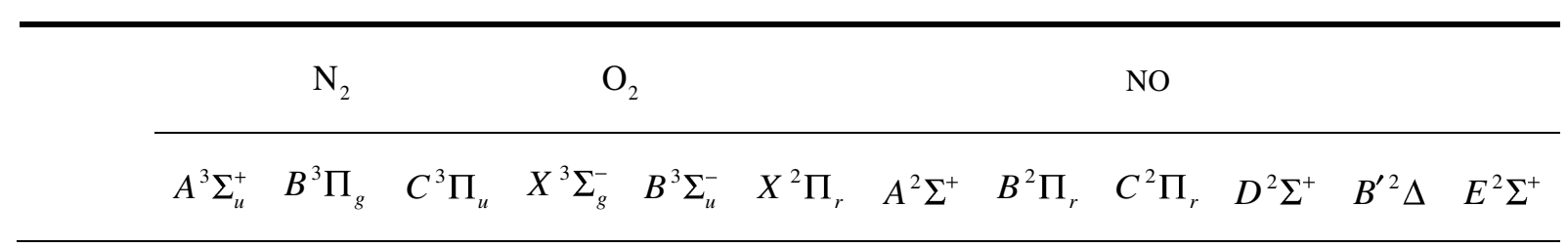




\begin{tabular}{ccccccccccccc}
$I_{i}$, & 75427 & 66012 & 36494 & 98401 & 48608 & 76800 & 32834 & 30867 & 24620 & 23715 & 16435 & 16171 \\
$\mathrm{~cm}^{-1}$ & & & & & & & & & & & & \\
$\omega_{i}$ & 1.21 & 1.29 & 1.73 & 1.06 & 1.50 & 1.20 & 1.83 & 1.89 & 2.11 & 2.15 & 2.58 & 2.61 \\
\hline
\end{tabular}

Assuming that the electronic, vibrational and rotational energy modes of diatomic molecules are independent, the energy of the $i$ th level can be expressed as [13]

$$
E(e, v, J)=E(e)+G(v)+F(J)
$$

where $e, v, J$ are the quantum number of electronic, vibrational and rotational energy levels. In this case, the degeneracy of the $i$ th level is

$$
g_{i}=g_{e} \sum_{v, J} g_{J} \exp \left(-\frac{G(v)}{k T_{v}}-\frac{F(J)}{k T_{r}}\right)
$$

To investigate the difference between the nonequilibrium and equilibrium radiation of diatomic molecules, we reconsider the air plasma cell listed in Table 2. We only calculate $\mathrm{N}_{2}$ as an example. The first positive system $\left(B^{3} \Pi_{g}-A^{3} \Sigma_{u}^{+}\right)$and the second positive system $\left(C^{3} \Pi_{u}-B^{3} \Pi_{g}\right)$ of $\mathrm{N}_{2}$ are studied.

Figure 2 shows the spectral emission of $\mathrm{N}_{2}$. Here, we only consider the bound-bound transitions, and the Voigt profile is used as the lineshape. The results in Fig. 2(a) is based on the multi-temperature (multi-T) model, where the population of electronic, vibrational, and rotational energy levels is assumed to be Boltzmannian distribution characterized by $T_{e}, T_{v}$, and $T_{r}$, respectively. The results in Fig. 2(b) is based on our method. Specifically, the population of electronic levels is calculated by the above-mentioned method, whereas the population of vibrational and rotational levels is assumed to be Boltzmannian distribution characterized by $T_{v}$ and $T_{r}$, respectively.
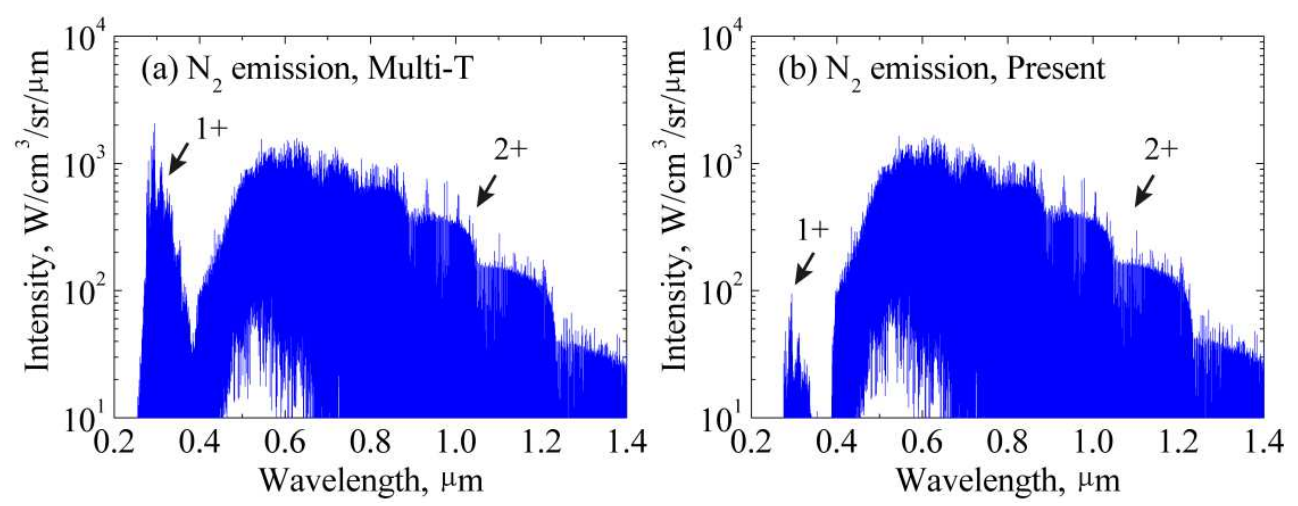

Fig. 2 Emission of $\mathrm{N}_{2}$ based on the multi-T model (a) and the present method.

Compared with Fig. 2(b), the intensity of the first positive system in Fig. 2(a) is significantly larger, while the intensity of the second positive system is slightly smaller. The results indicate that the multi-T model overestimate the emission of $\mathrm{N}_{2}$. The overestimation is attributed to the error in the population of 
electronic levels.

Based on our method, the calculated nonequilibrium coefficients are listed in Table 4. These nonequilibrium coefficients suggest that, compared with the population determined by the Saha-Boltzmann equation, the population of $B^{3} \Pi_{g}$ is 5.89 times larger, whereas the population of $C^{3} \Pi_{u}$ is 0.25 times smaller.

Table 4 The nonequilibrium coefficients.

\begin{tabular}{cc} 
Levels & $b_{i}$ \\
\hline$B^{3} \Pi_{g}$ & 5.89 \\
$C^{3} \Pi_{u}$ & 0.25 \\
\hline
\end{tabular}

\section{Application in hypersonic flow}

To verify the application of the present method in hypersonic nonequilibrium air plasmas, we chose to study the cell conditions listed in Ref. [13]. We did not discuss the equilibrium states, but focus the attention on the nonequilibrium AVCO R-156 experiments.

Based on the nonequilibrium cell condition in Ref. [13], we calculated the fine spectra with a high resolution $(0.001 \mathrm{~nm})$. The results are shown in Fig. 3(a). Here, the bound-bound, bond-free, and free-free transitions of particles are considered. It is seen that atomic lines are dominant due to the high temperature.

Regarding to the molecular bands, the emission of $\mathrm{NO}$ and $\mathrm{O}_{2}$ makes the spectral intensity below $200 \mathrm{~nm}$ relatively strong. Meanwhile, the peak intensity at $\sim 400 \mathrm{~nm}$ is mainly due to the emission of $\mathrm{N}_{2}^{+}$. However, the emission of $\mathrm{N}_{2}$ is relatively weak.
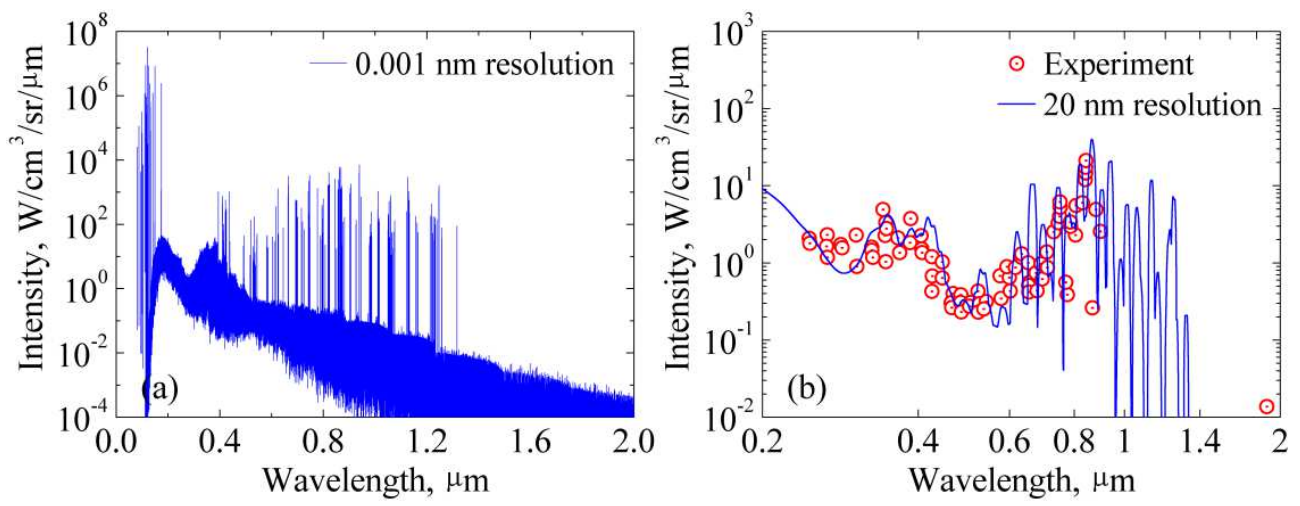

Fig. 3 Emission of the AVCO R-156 nonequilibrium air plasma. (a) The calculated fine spectra. (b) A comparison between our simulation and the measured data.

Figure 3(b) shows a comparison between our simulation and the nonequilibrium AVCO R-156 experimental spectra. The resolution of the calculated spectrum is converted to $20 \mathrm{~nm}$, which is the same as 
the resolution of the measured data. It is clearly seen that our results are in good agreement with the experimental data. This indicates that the present method is reasonable, and has a good accuracy. In the spectral region of 650-700 nm, there is a deviation between our results and the experimental data. It can be attributed to the following two reasons. One is that the transition probabilities related to the atomic lines within $663-665 \mathrm{~nm}$ are inaccurate to a certain extent, as Table 5 shows [26]. The other is that, owing to the lack of transition probabilities, the spontaneous transitions from the upper levels to the 12th level are not included in the present calculation.

Table 5 Transition probabilities related to atomic lines within 663-665 nm.

\begin{tabular}{ccc}
\hline Wavelength, $\AA$ & $A_{k i}, 10^{8} \mathrm{~s}^{-1}$ & Possible deviation \\
\hline 6644.96 & 0.0311 & $25 \%$ \\
6653.46 & 0.0244 & $25 \%$ \\
6656.51 & 0.0193 & $25 \%$ \\
6636.94 & 0.0125 & $25 \%$ \\
6646.51 & 0.0194 & $25 \%$ \\
\hline
\end{tabular}

Since it is not necessary to solve the rate equations of energy level populations, the present method is simpler than most of QSS models including the NEQAIR code. It is applicable for the 11-species air plasmas that contains $\mathrm{N}, \mathrm{N}^{+}, \mathrm{O}, \mathrm{O}^{+}, \mathrm{N}_{2}, \mathrm{~N}_{2}^{+}, \mathrm{O}_{2}, \mathrm{O}_{2}^{+}, \mathrm{NO}, \mathrm{NO}^{+}$and free electrons. In fact, if the corresponding gas components are added, this method is also suitable for a variety of hypersonic applications.

\section{Conclusions}

We developed a simplified method for calculating spectral emission of nonequilibrium air plasmas. The method introduces nonequilibrium coefficients into the population of each energy level governed by the Saha-Boltzmann equation. By considering the three significant radiative processes, the nonequilibrium coefficients can be calculated. Thus, the nonequilibrium populations can be obtained. For diatomic molecules, an approach to the determination of the radiative recombination coefficient and the energy level degeneracy is presented. Based on the approach, we can also calculate the nonequilibrium coefficients. A typical air plasma cell is chosen to investigate the difference between the nonequilibrium and equilibrium emission of atoms and molecules. It is suggested that the multi-T model overestimate the spectral emission in comparison with our results. Application of the present method to the nonequilibrium AVCO R-156 flowfield was shown. The results indicate that the present method agrees well with the experimental data. This work offers a simple method to the calculation of nonequilibrium air plasma radiation, which will be useful in hypersonic engineering.

\section{Acknowledgements}

This work is supported by the National Numerical Windtunnel (NNW2019ZT3-B07) and the National Natural Science Foundation of China (11674395 ). The authors would like to thank Prof. Junbo Yang and Prof. Juncai Yang for their helpful work. The authors are thankful to the reviewers for their valuable comments to improve the quality of the manuscript. 


\section{Funding}

The National Numerical Windtunnel (NNW2019ZT3-B07) and the National Natural Science Foundation of China (11674395).

\section{Availability of data and materials}

Data supporting the results of this article can be found, including data generated during the study. According to reasonable requests, the minimal datasets necessary to interpret and replicate the results of this article can be obtained from the corresponding author.

\section{Authors' contributions}

$\mathrm{XH}$ proposed the method for calculating the bound electronic energy level population of atoms and molecules with help of TJ. CG collected the quantum data of atoms and molecules. XH performed the calculation and data analysis with help of CG and TJ. XH and CG wrote the manuscript. TJ reviewed and processed the manuscript. All authors read and approved the final manuscript.

\section{Competing interests}

The authors declare that they have no competing interests.

\section{References}

[1] Surzhikov S T, Radiative-collisional models in non-equilibrium aerothermodynamics of entry probes, Journal of Heat Transfer, 134, 031002 (2012).

[2] Park C, Nonequilibrium hypersonic aerothermodynamics, New York: Wiley Press, 1990.

[3] Arnold J O, Whiting E, Lyle G C, Line by line calculation of spectra from diatomic molecules and atoms assuming a Voigt line profile, Journal of Quantitative Spectroscopy and Radiative Transfer, 9, 775-798 (1969).

[4] Dong S K, Ma Y, Tan H P, Modeling of high-temperature air species nonequilibrium spectral radiation properties, Journal of Thermophysics and Heat Transfer, 22(2), 301-305 (2008).

[5] Shang J S, Surzhikov S T, Simulating nonequilibrium flow for ablative earth reentry, Journal of Spacecraft and Rockets, 47(5), 806-815 (2010).

[6] Fujita K, Sato S, Takashi A T, Experimental investigation of air radiation from behind a strong shock wave, Journal of Thermophysics and Heat Transfer, 16(1), 77-82 (2002).

[7] Park C, Nonequilibrium air radiation (NEQAIR) program: user's manual, In: NASA Report No. 1985-30780, 1985.

[8] Liu Y, Prabhu D, Trumble K A, Saunders D, Jenniskens P, Radiation modeling for the reentry of the Stardust sample return capsule, Journal of Spacecraft and Rockets, 47(5), 741-752 (2010).

[9] Ozawa T, Modest M F, Levin D A, Spectral module for photon monte carlo calculations in hypersonic nonequilibrium radiation, Journal of Heat Transfer, 132, 023406 (2010).

[10]Bansal A, Modest M F, Levin D A, Multi-scale k-distribution model for gas mixtures in hypersonic nonequilibrium flows, Journal of Quantitative Spectroscopy and Radiative Transfer, 112(12), 1213-1221 (2011). 
[11]Landshoff R K M, Magee J L, Thermal radiation phenomena, I: radiative properties of air, New York: Plenum Publishing Corporation, 1969.

[12] Laux C O, Optical diagnostics and radiative emission of air plasmas, Doctor of Philosophy Thesis, Stanford University, 1993.

[13] Moreau S, Laux C O, Chapman D R, MacCormack R W, A more accurate nonequilibrium air radiation code: NEQAIR second generation, In: AIAA Paper No. 1992-2968, 23rd Plasmadynamics and Laser Conference, Nashville, TN, 1992.

[14] Whiting E, Park C, Liu Y, Arnold J, Paterson J, NEQAIR96, nonequilibrium and equilibrium radiative transport and spectra program: user's manual, NASA/Ames Research Center, Moffett Field, CA, NASA Reference Publication 1389, 1996.

[15] Bansal A, Modest M F, Levinz D A, Correlated-k distribution method for atomic radiation in hypersonic nonequilibrium flows, In: AIAA Paper No. 2009-1027, 47th AIAA Aerospace Sciences Meeting and Exhibit, Orlando, Florida, 2009.

[16]He X, Chang S-L, Dai S-A, Yang J-C, Simulation of radiative transfer in nonequilibrium plasmas containing $\mathrm{N}$ and $\mathrm{O}$ species based on the approximate collision-radiative method, Chinese Physics Letters, 30(11), 114401 (2013).

[17] He X, Dang W-H, Jia H-H, Yin H-W, Zhang H-L, Chang S-L, Yang J-C, Analysis of atomic electronic excitation in nonequilibrium air plasmas, Chinese Physics Letters, 31(9), 095204 (2014).

[18] Laux C O, Moreau S, Kruger C, Experimental study and improved modeling of high-temperature air radiation, In: AIAA Paper No. 1992-2969, 23rd Plasmadynamics and Laser Conference, Nashville, TN, 1992.

[19] Park C, Calculation of nonequilibrium radiation in the flight regimes of aeroassisted orbital transfer vehicles, Thermal Design of Aeroassisted Orbital Transfer Vehicles, 96, 395-418 (1985).

[20] Park C, Review of chemical-kinetic problems of future NASA mission, I: earth entries, Journal of Spacecraft and Rockets, 7(3), 385-398 (1993).

[21] Tauber M E, Palmer G E, Yang L, Earth atmospheric entry studies for manned Mars mission, Journal of Thermophysics and Heat Transfer, 6, 193-199 (1992).

[22] Shang J S, Surzhikov S T. Simulating Stardust earth reentry with radiation heat transfer, Journal of Spacecraft and Rockets, 48(3), 385-396 (2011).

[23] Lamet J M, Babou Y, Rivière P, Perrin M Y, Soufiani A, Radiative transfer in gases under thermal and chemical nonequilibrium conditions: application to earth atmospheric re-entry, Journal of Quantitative Spectroscopy and Radiative Transfer, 109, 235-244 (2008).

[24] Peach G, Continuous absorption coefficients for non-hydrogenic atoms, Memoirs of the Royal Astronomical Society, 73, 1-123 (1970).

[25] Herzberg G, Molecular spectra and molecular structure, I: spectra of diatomic molecules, Beijing: Science Press, 1983.

[26] Wiese W L, Smith M W, Glennon B M, Atomic transition probabilities, I: hydrogen through neon, National Standard Reference Data Series, National Bureau of Standards 4, 1966. 
Figures
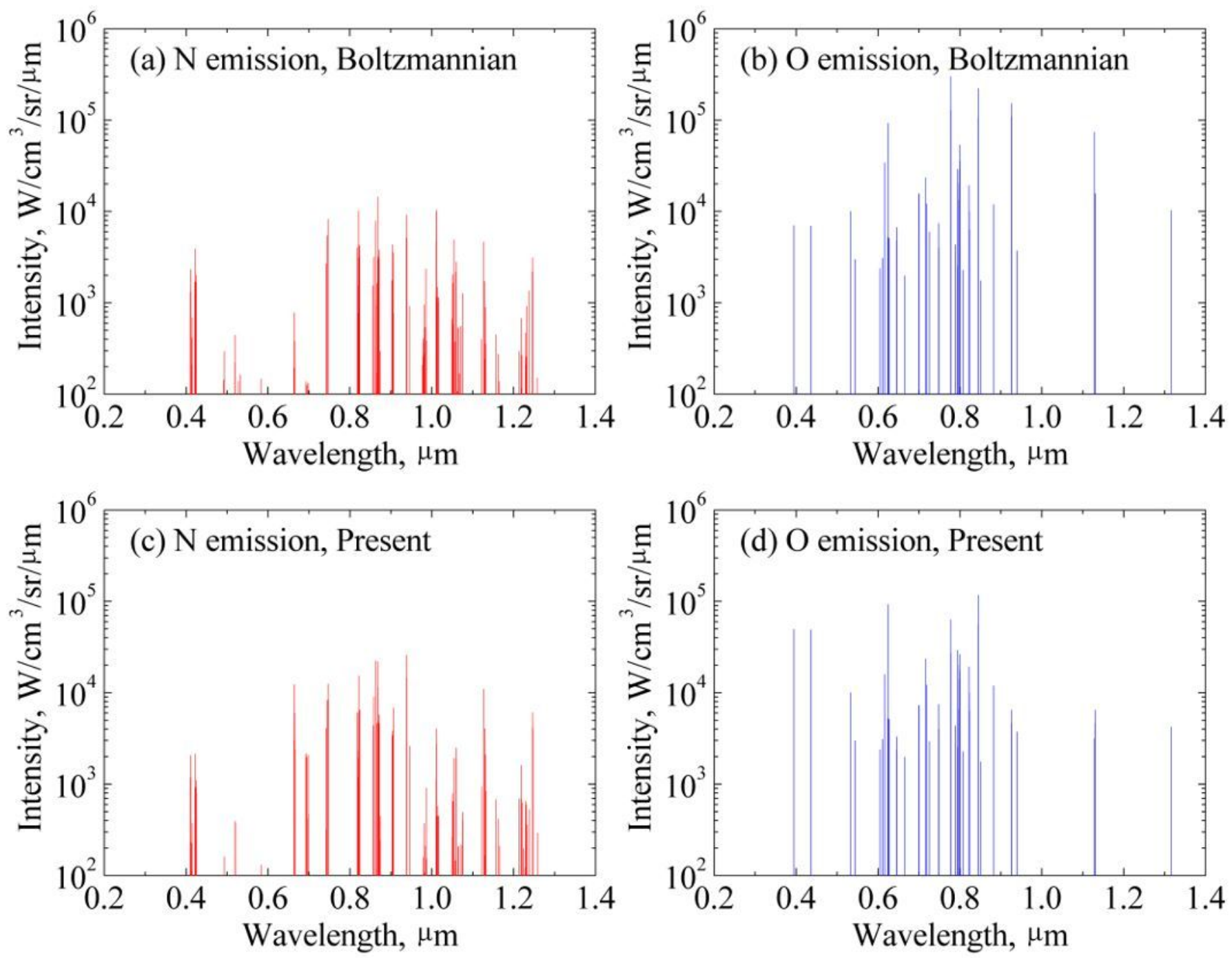

Figure 1

spectral emission of $\mathrm{N}(\mathrm{a})$ and $\mathrm{O}(\mathrm{b})$ assuming a Boltzmannian distribution with Te. Nonequilibrium emission of $\mathrm{N}(\mathrm{c})$ and $\mathrm{O}$ (d) using the present method. 

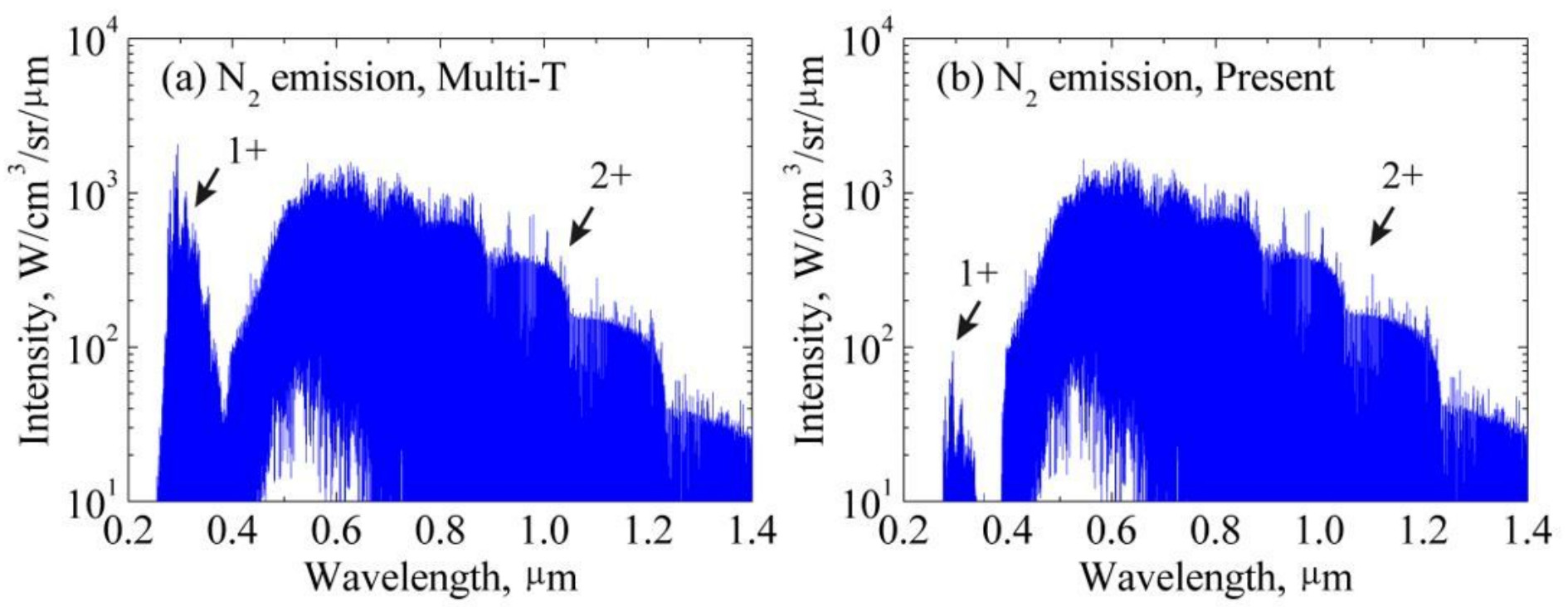

Figure 2

Emission of N2 based on the multi-T model (a) and the present method.
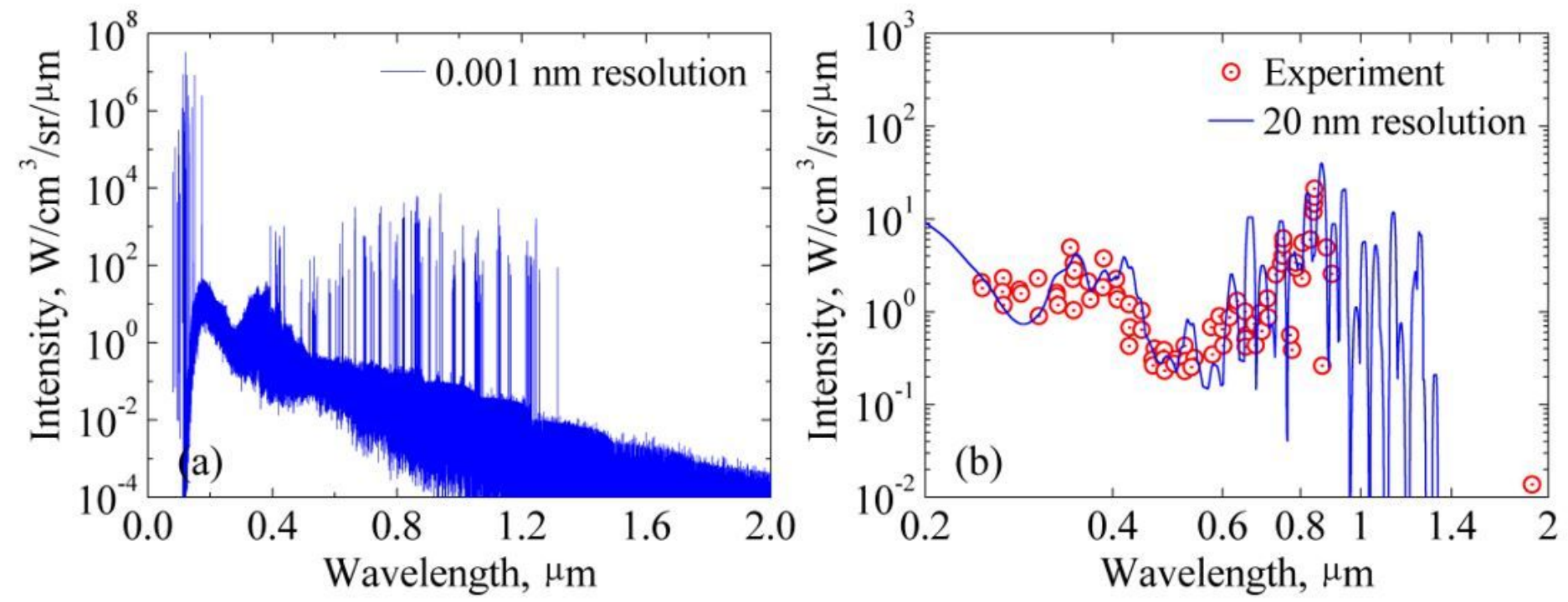

Figure 3

Emission of the AVCO R-156 nonequilibrium air plasma. (a) The calculated fine spectra. (b) A comparison between our simulation and the measured data. 